\title{
Aceitabilidade de pão fortificado com ferro microencapsulado por crianças de creches das regiões sul e leste da cidade de São Paulo'
}

\author{
Acceptability of bread fortified with microencapsulated \\ iron by children of daycare centers in the south \\ and east regions of São Paulo city, Brazil
}

Teresinha Stumpf SOUTO2

Anne Lise Dias BRASIL ${ }^{2}$

José Augusto de Aguiar Carrazedo TADDEI²

\section{R E S U M O}

\section{Objetivo}

Avaliar a aceitabilidade de um pão tipo bisnaguinha enriquecido com ferro microencapsulado por crianças freqüentadoras de creches, de acordo com sexo, faixa etária, estado nutricional, presença de anemia, tempo de estocagem e momento do experimento (inicial, meio e final).

\section{Métodos}

Ensaio clínico randomizado duplo-cego com 382 crianças de 2 a 6 anos de idade, matriculadas em quatro creches filantrópicas do município de São Paulo. As crianças de duas creches receberam no café da manhã, de segunda a sexta-feira, o pão fortificado com ferro microencapsulado e as das outras duas, pão idêntico, porém sem ferro, durante 120 dias. A ingestão foi anotada diariamente em planilhas individuais. Os testes estatísticos utilizados foram o qui-quadrado, o teste $t$ de Student, a análise de variância e a análise de covariância.

\section{Resultados}

O consumo médio semanal de pães foi significantemente menor nas crianças das creches expostas $(1,17)$ do que nas creches não expostas $(1,56)$ e a variabilidade de aceitação foi maior nas creches não expostas. A ingestão foi significantemente menor pelas crianças das creches expostas em todas as variáveis estudadas, exceto nas faixas etárias menores de 36 meses e de 36 a 47 meses.

\footnotetext{
1 Artigo elaborado a partir da dissertação de T.S. SOUTO, intitulada "Aceitação de pão enriquecido com ferro microencapsulado por crianças de creches". Universidade Federal de São Paulo; 2006.

2 Universidade Federal de São Paulo, Escola Paulista de Medicina, Departamento de Pediatria. R. Loefgreen, 1647, 04040-032, São Paulo, SP, Brasil. Correspondência para/Correspondence to: J.A.A.C. TADDEI. E-mail: <taddei.dped@epm.br>.
} 


\section{Conclusão}

Apesar de a aceitação pelas crianças do pão fortificado com ferro ter sido significantemente menor do que ao pão sem ferro, a aceitação nos dois grupos mostra que este pão pode ser alternativa viável para a fortificação de alimentos na prevenção da anemia ferropriva em crianças de creches.

Termos de indexação: Alimentos fortificados. Anemia ferropriva. Creches. Pré-escolar.

\section{A B S T R A C T}

\section{Objective}

To evaluate the acceptance of a new food, a mini-roll enriched with microencapsulated iron sulfate, among preschool-aged children stratified by sex, age group, nutritional status, presence of iron-deficiency anemia, length of storage, and stage of trial (early, middle, late).

\section{Methods}

Double blind randomized clinical trial with 382 children aged 2 to 6 years enrolled in 4 not-for-profit daycare centers in the city of São Paulo, Brazil. Children in two daycare centers received the mini-rolls fortified with microencapsulated iron in breakfast from Monday to Friday (exposed daycare centers). Children in the other 2 daycare centers received regular, yet identical, mini-rolls in the same manner. Observation lasted 120 days. Acceptance was noted daily on specific log-sheets. Data were compared using the chi-square and Student's t tests, analysis of variance and analysis of covariance.

\section{Results}

Mean weekly consumption of mini-rolls was lower in exposed daycare centers (1.17) than in unexposed centers (1.54). Variance was greater in unexposed daycare centers. Acceptance was lower among children in exposed daycare centers in all stratified variable categories, except among children younger than 36 months or aged between 36 and 47 months.

\section{Conclusion}

Although the children's acceptance of the rolls fortified with iron was significantly lower than that of rolls without iron fortification, this type of food might be a viable alternative in the prevention of iron-deficiency anemia in children of daycare centers.

Indexing terms: Food, fortified. Anemia, iron-deficiency. Child Day Care Centers. Child, preschool.

\section{N T R O D U Ç Ã O}

A anemia nutricional foi definida pela Organização Mundial de Saúde (OMS) como uma situação na qual a concentração de hemoglobina nos glóbulos vermelhos é anormalmente baixa, em conseqüência da carência de um ou mais nutrientes essenciais, qualquer que seja a origem desta. Quando esse quadro ocorre devido à deficiência de ferro é denominada anemia ferropriva, sendo o tipo mais comum na infância e de grande relevância não só nos paises em desenvolvimento, como naqueles altamente industrializados ${ }^{1}$.

A OMS estima que dois bilhões de pessoas no mundo sejam ferropênicas e que, nos países com alta prevalência de anemia, exista, para cada indivíduo anêmico, mais um outro com deficiência de ferro ${ }^{1}$.

A carência nutricional de ferro é indicada como um dos 10 fatores de risco para doenças, incapacidade e morte no mundo de hoje. Tem repercussões negativas tanto no bem-estar dos indivíduos, como na produtividade da sociedade. Em relação à anemia ferropriva, principalmente nas crianças em idade pré-escolar, quanto maior a gravidade, maior a chance de atraso no desenvolvimento mental e motor com conseqüências em seu desenvolvimento intelectual futuro ${ }^{1-3}$.

No Brasil houve redução na prevalência da desnutrição, contudo a anemia ferropriva vem aumentando progressivamente nas últimas 
décadas. Dados do Ministério da Saúde revelaram que $30 \%$ das gestantes e $50 \%$ das crianças menores de dois anos no Brasil apresentavam anemia. No Estado de São Paulo, os estudos epidemiológicos sobre anemia, em crianças menores de cinco anos, mostraram aumento de mais de $100 \%$ nas últimas duas décadas, inclusive entre a população de melhor poder aquisitivo ${ }^{4,5}$.

Diante desse quadro, três estratégias são recomendadas para corrigir a deficiência nutricional de ferro, isoladamente ou combinando-se umas com as outras. São elas, a modificação dietética para melhorar o valor nutricional dos alimentos e a biodisponibilidade do ferro, a suplementação medicamentosa e a fortificação de alimentos ${ }^{6}$.

A fortificação de alimentos é mundialmente considerada como a solução mais prática e de melhor custo-benefício, principalmente para regiões, nas quais há grande prevalência dessa carência nutricional ${ }^{1,3,7}$.

Alguns critérios são necessários para a escolha dos alimentos a serem fortificados, tais como, consumo por toda a população-alvo, pequena variação per capita no consumo semanal, não ocorrência de alterações nas características organolépticas do produto, boa aceitabilidade, biodisponibilidade do nutriente no alimento, viabilidade econômica, razoável segurança frente ao risco de ingestão excessiva e estabilidade sob condições-padrão de armazenamento ${ }^{8,9}$.

Porém, embora vários compostos à base de ferro possam ser utilizados na fortificação, há problemas técnicos na seleção desses componentes. Os compostos de biodisponibilidade relativamente alta, como o sulfato ferroso, com freqüência, provocam alterações organolépticas inaceitáveis, enquanto que outros compostos mais aceitáveis são pouco absorvidos ${ }^{10}$.

Por essa razão, além de estudar formas alternativas de compostos de ferro, foi desenvolvida a técnica de microencapsulação que, ao isolar o ferro, mascara seu sabor, reduz a reatividade com outros componentes da dieta e controla a sua liberação em áreas do trato gastrintestinal, permitindo sua melhor absorção ${ }^{11}$.
A aceitabilidade de um novo alimento adquire importância prática já que, particularmente, na faixa etária entre dois e cinco anos de idade, é comum encontrar crianças que hesitam em provar ou que rejeitam novos alimentos, situação essa definida como neofobia alimentar. Os fatores mais importantes para que um novo alimento seja aceito são o contexto social no qual esse alimento é oferecido, suas repetidas exposições e as conseqüências de sua ingestão ${ }^{12-15}$.

Pesquisas com pré-escolares de dois a cinco anos demonstraram que são necessárias de 8 a 10 ou até mesmo 15 exposições a um novo alimento para diminuir a resposta neofóbica e aumentar sua aceitação ${ }^{12,16}$.

A forma como alguns autores avaliaram a aceitabilidade do alimento fortificado é bastante variável. Zlotkin et al. ${ }^{6}$ questionaram as crecheiras quanto à aceitabilidade de um suplemento em pó contendo ferro misturado à alimentação usual de crianças; em Bangladesh, os mesmos autores perguntaram às mães qual a percepção sobre a aceitação desse produto por parte de seus filhos. Sari et al. ${ }^{17}$ avaliaram a aceitabilidade de balas fortificadas por questões dirigidas às mães e às próprias crianças. Silva ${ }^{18}$ mediu a aceitação de alimentos fortificados com ferro por meio de escala hedônica em adultos, enquanto que Paula \& Fisberg ${ }^{19}$ referiram como excelente a aceitação de açúcar fortificado, em suco de laranja, por crianças de 10 a 48 meses de idade.

Apesar de existirem várias pesquisas com alimentos fortificados com ferro para a prevenção e o tratamento da anemia ferropriva, poucas publicaram detalhadamente a forma de avaliação da aceitabilidade por parte das crianças, tornandose relevante a realização desta pesquisa.

O objetivo do estudo foi o de avaliar a aceitabilidade de um pão enriquecido com ferro microencapsulado por crianças de creches, de acordo com sexo, faixa etária, estado nutricional, presença de anemia, tempo de estocagem e momento do experimento. 


\section{MÉ T O D O S}

Para efetivar o estudo foram produzidos pães tipo bisnaguinha com farinha de trigo enriquecida com sulfato ferroso microencapsulado com alginato de sódio, desenvolvido pelo Instituto de Pesquisas Tecnológicas do Estado de São Paulo (IPT-SP). O enriquecimento da farinha, a avaliação da sua estabilidade, a produção dos pães, o teste sensorial e o controle semanal de qualidade do produto, mediante amostras periódicas, foram realizados pelo Instituto de Tecnologia de Alimentos (ITAL) em Campinas, no Núcleo de Análises Físicas, Sensoriais e Estatística (LAFISE).

A biodisponibilidade do ferro foi avaliada pelo Laboratório de Nutrição-Minerais do Departamento de Nutrição Experimental da Faculdade de Ciências Farmacêuticas da Universidade de São Paulo (USP) e a quantificação de ferro no pão foi realizada pelo Laboratório de Pesquisa Clínica da Disciplina de Gastroenterologia do Departamento de Pediatria da Unifesp.

Cada pão tinha o peso médio de 20 gramas e continha $4 \mathrm{mg}$ de ferro, de acordo com a portaria $n^{\circ} 31$, de janeiro de 1988, da Agência Nacional de Vigilância Sanitária (ANVISA), que regulamenta o uso de alimentos fortificados ${ }^{20}$.

O ensaio clínico foi realizado em quatro creches do Município de São Paulo, mantidas pela Entidade Filantrópica Cruzada Pró-Infância, sendo duas na região Leste e duas na região Sul de São Paulo. Em cada região, uma creche foi randomicamente sorteada por um pesquisador externo para receber o pão fortificado (creche exposta) e outra, o não fortificado (creche não exposta).

O estudo foi do tipo ensaio clínico randomizado duplo cego, sendo realizado durante os meses de março a setembro de 2003, durante 120 dias.

O cálculo amostral foi realizado pelo programa Epi Info ${ }^{\circledR}$ versão 6.02, adotando erro $\alpha$ de 0,05 , erro $\beta$ de 0,20 e prevalência de anemia de $50 \%$ antes da intervenção, para detectar $15 \%$ de redução na prevalência de anemia no grupo exposto, ao término da intervenção, sendo estimada em 364 crianças (182 em cada grupo) ${ }^{21}$.
A amostra inicial do estudo foi formada por todas as 416 crianças matriculadas nas creches selecionadas, com idade compreendida entre 2 anos e 6 anos e 11 meses.

Houve perda de $8,2 \%$ da amostra inicial devida à não autorização de alguns responsáveis para participar na pesquisa, e ao absenteísmo igual ou maior a 20,0\% no período do estudo ou por dados incompletos, totalizando, no final do ensaio, 382 crianças.

Foi desenvolvida uma planilha para o controle diário e individual do consumo de pães e das faltas às creches. Esta planilha foi preenchida e supervisionada pelos pesquisadores envolvidos no ensaio, com a ajuda das crecheiras.

A padronização dos códigos para a marcação diária dos consumos dos pães foi a seguinte: $\mathrm{N}=$ não aceitou; $\mathrm{P}=$ aceitou $\mathrm{o}$ pão parcialmente; $\mathrm{T}=$ aceitou todo o pão; $\mathrm{TP}=$ aceitou um pão $\mathrm{e}$ mais uma metade, ou seja, comeu 1 pão e meio; $\mathrm{Rl}=$ aceitou o primeiro pão e repetiu uma vez, ou seja, comeu 2 pães inteiros; RIP= aceitou o primeiro e o segundo pão totalmente e aceitou o terceiro pão parcialmente, ou seja, comeu 2 pães e meio; $\mathrm{R} \Gamma=$ aceitou o primeiro pão e repetiu duas vezes, ou seja, comeu 3 pães e $F=$ faltou.

Os dados demográficos foram colhidos das fichas das crianças nas creches, a antropometria e a coleta de sangue por punção digital; foram realizadas no início, no meio e no final do estudo (semanas 1, 12 e 24).

O pão tipo bisnaguinha foi oferecido para todas as crianças, durante o café da manhã, com diferentes recheios (margarina, requeijão ou geléia de frutas), acompanhado de leite achocolatado ou café com leite. As educadoras foram orientadas para apenas oferecer os pãezinhos, sem pressionar as crianças para o seu consumo.

Os pães eram entregues em todas as creches uma vez por semana, às sextas-feiras, sendo consumidos apenas durante a semana seguinte. 
Para a antropometria as crianças foram pesadas com roupas íntimas e sem meias, em pé, em balança eletrônica digital, marca Kratos ${ }^{\circledR}$, tipo plataforma, com capacidade para $150 \mathrm{~kg}$ e escalas com divisões de 50 gramas. A balança foi regulada com peso padrão de $1 \mathrm{~kg}$ e zerada a cada pesagem. A medida da altura foi realizada com um estadiômetro portátil, marca Alturexata ${ }^{\circledR}$, contendo escala milimetrada e bloco móvel de metal.

Para a classificação nutricional foram utilizados os escores-Z dos índices peso para estatura (P/E) e estatura para idade (E/I), com pontos de corte de acordo com a World Health Organization ${ }^{22}$.

Para a dosagem dos níveis de hemoglobina (Hb) foi utilizado o hemoglobinômetro digital portátil de marca Hemocue-ß Hemoglobin Photometer $^{\circledR}$, que tem princípio na leitura fotométrica.

Para a classificação da anemia foram utilizados os pontos de corte de $\mathrm{Hb}<11,0 \mathrm{~g} / \mathrm{dL}$ para crianças até 59 meses e $\mathrm{Hb}<11,5 \mathrm{~g} / \mathrm{dL}$ para aqueles maiores ou com 60 meses, conforme a recomendação da Organização Mundial de Saúde' .

Os dados foram duplamente digitados e validados no software Epi info ${ }^{\circledR}$ versão 6.02. O cálculo dos escores-Z de P/E e E/l foi realizado pelo programa EPINUT.

O consumo médio semanal de pães foi calculado levando-se em conta o número total de pães consumidos na semana, dividido pelo número de dias em que as crianças freqüentaram as creches.

A homogeneidade entre os dois grupos (creches expostas e creches não expostas) quanto às variáveis sexo, faixa etária, estado nutricional e anemia, foi avaliada utilizando o teste quiquadrado de Pearson.

Para comparar a média geral de consumo de pães entre as creches expostas e as creches não expostas e em relação ao sexo, à faixa etária, ao estado nutricional e à anemia, usou-se o teste $t$ de Student para amostras independentes.
A análise de variância (ANOVA) com medidas repetidas foi utilizada para comparar o consumo de pão ao longo do tempo entre os dois grupos, considerando-se dois fatores: momento do experimento com três níveis (semana 1, semana 12 e semana 24) e os grupos com 2 níveis (creches expostas e creches não expostas).

Para investigar o consumo dos pães, levando-se em conta as demais variáveis (sexo, faixa etária, hemoglobina e escores-Z), foi ajustado um modelo de análise de covariância (ANCOVA), considerando como variável resposta a média geral de consumo de pães com 3 fatores (creche, sexo e faixa etária) e 3 covariáveis (hemoglobina, escore-Z de P/E e escore-Z de E/I). Neste caso, comparou-se a aceitabilidade dos pães pelas crianças das creches expostas e das creches não expostas, ajustando as médias pelo efeito das demais variáveis.

O programa estatístico utilizado para efetuar os cálculos foi o SPSS for Windows, versão 11.0 e o nível de significância adotado foi de 0,05.

Esse projeto foi aprovado pelo Comitê de Ética em Pesquisa da Universidade Federal de São Paulo, sob o número de protocolo 1009/2004, em outubro de 2004.

\section{RES U LT A D O S}

Os dados descritivos em relação ao sexo, à faixa etária, ao estado nutricional e à presença de anemia das crianças das creches expostas e creches não expostas no início do experimento (semana 1) estão apresentados na Tabela 1.

Quanto ao sexo, ao estado nutricional e à presença de anemia, os grupos de crianças de creches expostas e das não expostas eram semelhantes. Vale ressaltar que, em relação à faixa etária, encontrou-se maior prevalência de crianças com idade superior a 59 meses nas creches expostas $(p=0,014)$.

A Figura 1 mostra o consumo médio semanal de pães nas 24 semanas de observação. Pode-se notar que, em média, esse consumo foi 
Tabela 1. Distribuição das variáveis demográficas e nutricionais, segundo exposição ao pão enriquecido com ferro no início do experimento (semana 1). São Paulo (SP), 2003.

\begin{tabular}{|c|c|c|c|c|c|}
\hline \multirow{3}{*}{ Variáveis } & \multicolumn{4}{|c|}{ Creches } & \multirow{3}{*}{ Valor de $p$} \\
\hline & \multicolumn{2}{|c|}{ Expostos } & \multicolumn{2}{|c|}{$\begin{array}{c}\text { Não } \\
\text { expostos }\end{array}$} & \\
\hline & $n$ & $\%$ & $\mathrm{n}$ & $\%$ & \\
\hline \multicolumn{6}{|l|}{ Sexo } \\
\hline Masculino & 108 & 47,2 & 80 & 52,3 & 0,326 \\
\hline Feminino & 121 & 52,8 & 73 & 47,7 & \\
\hline \multicolumn{6}{|l|}{ Faixa etária } \\
\hline$<36$ meses & 55 & 24,0 & 30 & 19,6 & \\
\hline 36 a 47 meses & 42 & 18,3 & 42 & 27,4 & 0,014 \\
\hline 48 a 59 meses & 49 & 21,4 & 44 & 28,8 & \\
\hline$>59$ meses & 83 & 36,2 & 37 & 24,2 & \\
\hline \multicolumn{6}{|l|}{ Estado nutricional } \\
\hline Em vigilância para desnutrição & 19 & 8,3 & 10 & 6,5 & \\
\hline Eutróficos & 166 & 72,5 & 118 & 77,1 & 0,589 \\
\hline Sobrepeso + Obesidade & 44 & 19,2 & 25 & 16,3 & \\
\hline \multicolumn{6}{|l|}{ Estatura } \\
\hline $\begin{array}{l}\text { Baixa estatura + Vigilância } \\
\text { para baixa estatura }\end{array}$ & 57 & 24,9 & 31 & 20,3 & \\
\hline Estatura normal & 141 & 61,6 & 97 & 63,4 & 0,498 \\
\hline $\begin{array}{l}\text { Vigilância para alta estatura } \\
\text { ou alta estatura }\end{array}$ & 31 & 13,5 & 25 & 16,3 & \\
\hline \multicolumn{6}{|l|}{ Anemia } \\
\hline Não & 123 & 53,7 & 66 & 43,1 & 0,443 \\
\hline Sim & 106 & 46,3 & 87 & 56,9 & \\
\hline
\end{tabular}

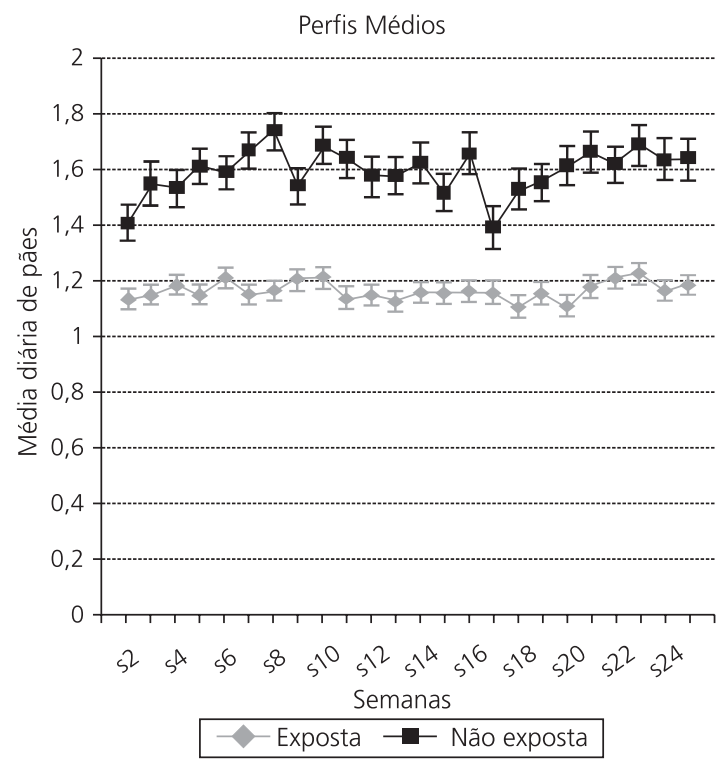

Figura 1. Consumo médio diário nas semanas 1 a 24, segundo creches expostas (pão enriquecido) e creches não expostas (pão não enriquecido). São Paulo (SP), 2003. significantemente menor nas creches expostas e também que a variabilidade da aceitação foi maior nas crianças das creches não expostas.

Foram realizadas comparações de aceitação do pão entre os grupos (creches expostas e creches não expostas) em 3 momentos: inicial (semana 1), meio (semana 12) e final (semana 24). Observou-se que houve diferença estatisticamente significante entre os grupos $(p<0,001)$, em todos os momentos de avaliação.

Como não houve grandes tendências de aumento ou diminuição de consumo ao longo do tempo, foi considerada a média geral, ou seja, a média diária de consumo de pães ao longo do período de observação.

A média geral de aceitação do pão pelas crianças nas creches expostas foi de 1,17 (Desvio-Padrão - $\mathrm{DP}=0,45$ ) e nas creches não expostas $1,56(D P=0,69)$. Verificou-se que a diferença foi estatisticamente significante $(p<0,001)$, ou seja, o consumo de pão foi, em média, menor entre as crianças das creches expostas. A diferença média foi de 0,4 pão (intervalo de confiança - IC $(95 \%)=[0,26 ; 0,51])$.

Na Tabela 2 verificou-se que houve diferenças nas médias de aceitação do pão pelas crianças de creches expostas e creches não expostas segundo o sexo, a faixa etária, o estado nutricional, a presença de anemia e o momento do experimento. O consumo nas creches expostas foi significantemente menor do que nas creches não expostas. Não se observou diferença significante apenas nas faixas etárias menores de 36 meses e de 36 a 47 meses de idade.

Também era de interesse da pesquisa avaliar a aceitação do pão de acordo com o tempo de estocagem, ou seja, comparar o consumo de pães no início e no fim da semana (segunda e sexta-feira) nas creches expostas e creches não expostas. Utilizando-se o teste estatístico ANOVA com medidas repetidas, foi observado que não houve diferença entre o consumo nas segundas e sextas $(p=0,440)$ para ambos os grupos. 
Tabela 2. Média e erros padrão (ep) da aceitabilidade de pães para as crianças freqüentadoras das creches expostas (pão enriquecido) e creches não expostas (pão não enriquecido), segundo variáveis demográficas, nutricionais e momento do experimento. São Paulo (SP), 2003.

\begin{tabular}{|c|c|c|c|c|c|}
\hline & \multicolumn{4}{|c|}{ Aceitabilidade } & \multirow{3}{*}{ Valor de $p$} \\
\hline & \multicolumn{2}{|c|}{ Creches expostas } & \multicolumn{2}{|c|}{ Creches não expostas } & \\
\hline & Media & (ep) & Media & (ep) & \\
\hline \multicolumn{6}{|l|}{ Sexo } \\
\hline Masculino & 1,18 & $(0,05)$ & 1,55 & $(0,08)$ & $<0,001$ \\
\hline Feminino & 1,15 & $(0,04)$ & 1,55 & $(0,07)$ & $<0,001$ \\
\hline \multicolumn{6}{|l|}{ Faixa etária } \\
\hline Menos de $36 \mathrm{~m}$ & 0,92 & $(0,05)$ & 1,02 & $(0,08)$ & 0,257 \\
\hline $36 \mathrm{~m}$ a $47 \mathrm{~m}$ & 1,04 & $(0,06)$ & 1,19 & $(0,10)$ & 0,208 \\
\hline $48 m$ a $59 m$ & 1,27 & $(0,06)$ & 1,80 & $(0,09)$ & $<0,001$ \\
\hline $60 \mathrm{~m}$ ou mais & 1,33 & $(0,05)$ & 2,09 & $(0,08)$ & $<0,001$ \\
\hline \multicolumn{6}{|l|}{ Estado nutricional (peso/estatura) } \\
\hline Desnutridos + vigilância para desnutrição & 1,08 & $(0,09)$ & 1,62 & $(0,21)$ & 0,034 \\
\hline Eutróficos & 1,16 & $(0,04)$ & 1,54 & $(0,07)$ & $<0,001$ \\
\hline Sobrepesos + obesos & 1,23 & $(0,07)$ & 1,56 & $(0,12)$ & 0,022 \\
\hline \multicolumn{6}{|l|}{ Anemia } \\
\hline Não & 1,24 & $(0,04)$ & 1,62 & $(0,08)$ & $<0,001$ \\
\hline Sim & 1,07 & $(0,04)$ & 1,49 & $(0,08)$ & $<0,001$ \\
\hline \multicolumn{6}{|l|}{ Momento do experimento } \\
\hline Inicial (semana 1) & 1,13 & $(0,04)$ & 1,40 & $(0,06)$ & $<0,001$ \\
\hline Meio (semana 12) & 1,13 & $(0,04)$ & 1,58 & $(0,07)$ & $<0,001$ \\
\hline Final (semana 24) & 1,18 & $(0,04)$ & 1,64 & $(0,07)$ & $<0,001$ \\
\hline
\end{tabular}

M: meses.

No modelo estatístico de análise de covariância (ANCOVA), que compara as médias de aceitação do pão nos dois grupos (creches expostas e creches não expostas) ajustadas para as outras variáveis, observou-se que não houve influência do sexo $(p=0,605)$, estado nutricional $(p=0,913)$ e escore-Z de E/I $(p=0,085)$.

Quando se utilizou o modelo ANCOVA, ajustando as médias pelo efeito das variáveis faixa etária e hemoglobina, verificou-se que as diferenças entre creches expostas e creches não expostas dependiam apenas da faixa etária.

Nos grupos de 48 a 59 meses e de 60 meses ou mais, o consumo médio de pão nas creches expostas foi significantemente menor do que nas creches não expostas $(p<0,001)$, enquanto que nas crianças menores de 36 meses $(p=0,3862)$ e entre 36 e 47 meses $(p=0,1318)$, não houve diferença (Figura 2).

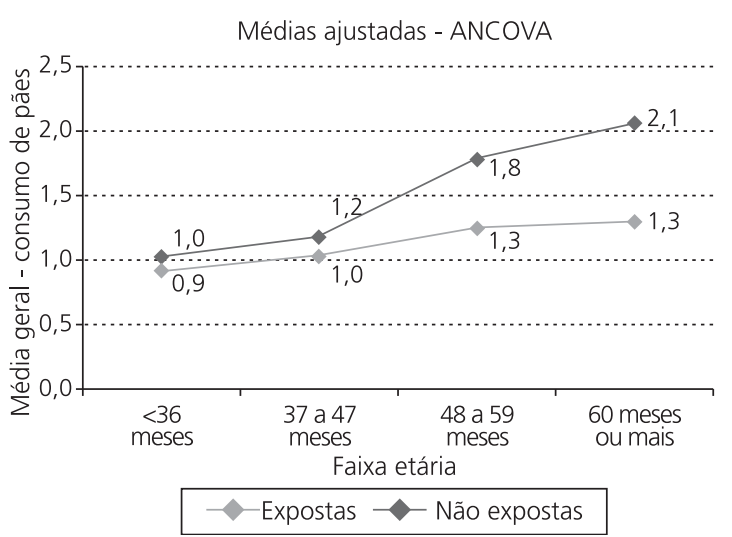

Figura 2. Aceitabilidade média de pães para crianças das creches expostas (pães enriquecidos com ferro) e creches não expostas (pão sem enriquecimento), segundo faixa etária (médias ajustadas para valores de hemoglobina utilizando Análise de Covariância - ANCOVA). São Paulo (SP), 2003.

D I S C U S S Ã O

Em todo o mundo há preocupação com a carência nutricional de ferro e com a busca de 
formas eficientes para superá-la, especialmente quando se trata de crianças. São contribuições que, de fato, vêm ampliando a discussão e permitindo, cada vez mais, o avanço de pesquisas sobre alimentos enriquecidos com ferro.

Os estudos com fortificação de alimentos na literatura científica não demonstram com detalhes a aceitabilidade das crianças.

Referências à aceitabilidade, partindo de observações não quantificadas, podem levar a erros de avaliação. Se não forem viabilizados métodos confiáveis de mensuração da ingestão, pode-se incorrer no risco de aceitar como verdadeira apenas a informação dos autores como sendo "exce-lente", "boa" ou "ótima".

Alguns trabalhos referem o volume ou a quantidade consumida do alimento fortificado de uma forma estimada, por meio de amostragem ou por média de ingestão ${ }^{23-26}$.

Vários trabalhos com alimentos fortificados para crianças no Brasil referiram boa aceitabilidade, porém não realizaram grupo-controle como, por exemplo, os estudos com água fortificada de Beinner et al. ${ }^{23}$ e de Dutra de Oliveira et al. ${ }^{27}$. Em ambos os estudos, as crianças consumiam a água fortificada ad libitum (cerca de $500 \mathrm{~mL} /$ dia), porém essa era a única fonte de água disponível. A ausência da oferta de água sem adição de ferro não permitiu a comparação adequada da aceitabilidade da água fortificada.

Em outras pesquisas utilizando leite, pão, biscoitos e suco fortificados para crianças, a aceitação foi referida como boa, mas os alimentos também não foram comparados com similares não fortificados, o que, certamente, daria maior credibilidade aos resultados, quanto à efetividade na prevenção e no tratamento da deficiência nutricional de ferro $24,26,28$.

Dutra et al.29,30, após alguns anos, realizaram outros estudos comparando a ingestão de água fortificada com ferro e ácido ascórbico e de água não fortificada, observaram que não havia diferenças na aceitabilidade entre os dois grupos.
Os resultados encontrados diferem dos do presente trabalho, no qual se observou menor aceitação do alimento enriquecido com ferro.

Na Indonésia, Sari et al. ${ }^{17}$ realizaram um estudo duplo-cego, oferecendo balas fortificadas com ferro às crianças de creche (4 a 6 anos de idade) e observaram a aceitabilidade. Apesar de o desenho da pesquisa ser adequado, uma eventual recomendação desta guloseima altamente cariogênica, é, no mínimo, questionável, principalmente para crianças. A idade pré-escolar é um momento chave para a introdução e a internalização de hábitos alimentares saudáveis que tendem a se perpetuar na idade adulta. Compreende-se a intenção dos pesquisadores ao utilizar as balas como veículos de fortificação, porém, guloseimas já são amplamente consumidas pela população de baixa renda, sendo desnecessário e maléfico o incentivo ao seu consumo.

Por se tratar de um estudo duplo-cego, a atual pesquisa tem importância científica, pois permitiu a comparação da aceitabilidade entre o alimento fortificado e o não fortificado, evitando o viés de aferição do observador. Por meio do registro individual diário, durante todo o período do estudo, foi possível demonstrar a ingestão do pão com rigor e método.

No presente estudo, embora o consumo médio de pão nos dois grupos tenha sido adequado para a faixa etária, observou-se que foi menor pelas crianças das creches expostas, provavelmente, pela alteração de sabor que a fortificação com ferro determina, mesmo quando microencapsulado.

Na análise da aceitabilidade do pão segundo o sexo, a faixa etária, o estado nutricional, a presença de anemia e o momento do experimento, observou-se ser significantemente menor nas creches expostas do que nas creches não expostas, exceto para as faixas etárias menores. Esse fato mostra que a ingestão do pão foi menor nas creches expostas, independentemente das variáveis observadas. Entretanto, a separação por 
faixas etárias permitiu observar diferenças na aceitabilidade nas diversas idades estudadas. Não foi encontrada nenhuma pesquisa na literatura científica que observasse a ingestão de um alimento fortificado de acordo com essas variáveis, não permitindo comparações.

Quando corrigida a média de aceitação das crianças nas faixas etárias menores de 48 meses, não se observou diferença estatisticamente significante no consumo do pão. Provavelmente as crianças menores, pela menor diversidade de sabores conhecidos, aceitaram com maior facilidade um sabor novo, oferecido repetidas vezes, enquanto que, as maiores, com mais experiências sensoriais, apresentaram mais resistência em aceitar um alimento que não é conhecido ${ }^{14,31}$.

Birch $^{31}$ afirmava que havia algumas diferenças no comportamento alimentar de acordo com a faixa etária, sendo as preferências das crianças de 3 anos mais influenciadas pelo modelo dos colegas do que as das crianças de 4 anos.

A diferença na aceitação dos pães encontrada na faixa etária remete aos resultados de Birch, pois, possivelmente, as crianças menores de 48 meses nos dois grupos (expostos e não expostos) apresentaram ingestão semelhante, agindo em função do grupo, por imitação ${ }^{13,31}$.

A literatura mostra que a ingestão de um alimento novo é incentivada quando há a presença de um adulto familiar ingerindo o mesmo alimento que a criança. Adessi et al. ${ }^{13}$ verificaram que as influências sociais são os fatores mais importantes na aceitação e no consumo de novos alimentos por crianças. São também relatados como fatores positivos a ausência de pressão por parte dos adultos, a verbalização do desejo de que a criança experimente ou apenas um olhar de aprovação ${ }^{15}$.

A criança deve experimentar várias vezes para reconhecer o novo sabor e o aceitar ${ }^{14,31}$. Apesar de ser esperada que a aceitação do pão aumentasse à medida que o experimento progredisse, não se encontrou diferença significante nas médias de aceitação do pão, no início, no meio e no final do estudo. Sabe-se que esse tipo de pesquisa com avaliação diária, por um longo período de tempo, é importante, pois a capacidade de aceitação de um novo alimento aumenta pelas exposições repetidas.

O pão tipo bisnaguinha foi escolhido para a fortificação com ferro, pelo fato de ser um alimento que costuma ser apreciado pelas crianças e ter pequenas dimensões, portanto, ser bem aceito pelos pré-escolares.

Quando se fala em fortificação com ferro, há de considerar que o alimento fortificado atinja a população alvo realmente. Para as crianças de até 24 meses de idade, o leite seria o alimento mais próprio a ser fortificado, já que a dieta é predominantemente láctea nessa faixa etária, apesar de ser estímulo ao desmame. Já as crianças maiores podem beneficiar-se de alimentos com cereais fortificados, já que os ingerem em quantidades significativas ${ }^{7,24}$.

As críticas de que a fortificação da farinha não iria atingir as crianças menores nem sempre são verdadeiras, pois se observou aceitação dos pães, fortificados ou não, também nas idades de 24 a 47 meses, que são de grande risco para a anemia ferropriva.

Acredita-se que atuações ativas e estimulantes dos professores, desde programas de educação nutricional até mudanças na postura dos educadores, como a realização de suas refeições junto às crianças, sejam decisivas na incorporação de novos alimentos fortificados com ferro no hábito alimentar dos pré-escolares.

É necessário que mais pesquisas se comprometam a avaliar a aceitabilidade do alimento fortificado de forma adequada.

Concluiu-se que o pão fortificado com sulfato ferroso microencapsulado, apesar de apresentar diferença de sabor em relação ao pão não fortificado, observada pelas crianças, teve boa aceitabilidade, principalmente pelas menores de 48 meses. Portanto, este alimento pode ser uma alternativa viável na fortificação de alimentos para o controle da deficiência de ferro em crianças de creches. 


\section{COLABORADORES}

T.S. SOUTO foi responsável pela coleta, pela análise de dados e pela redação do artigo. A.L.D. BRASIL foi idealizadora do projeto, responsável pela elaboração e compilação dos dados e pela redação final do artigo. J.A.A.C. TADDEl foi responsável pela supervisão em todas as etapas da pesquisa.

\section{REFERÊ NCIAS}

1. World Health Organization. Iron deficiency anaemia assessment, prevention and control: a guide for programme managers. WHO/NHD/01.3. Geneva: WHO; 2001.

2. Lozoff B, Jimenez E, Hagen J, Mollen E, Wolf AW. Poorer behavioral and developmental outcome more than 10 years after treatment for iron deficiency in infancy. Pediatrics. 2000; 105(4): E51.

3. Lynch SR. The impact of iron fortification on nutritional anaemia. Best Pract Res Clin Haematol. 2005; 18(2):333-46.

4. Brasil. Ministério da Saúde. Compromisso social para redução da anemia por carência de ferro no Brasil. Brasília: Ministério da Saúde; 1999.

5. Monteiro CA, Szarfarc SC, Mondini L. Tendência secular da anemia na infância na cidade de São Paulo (1984-1996). Rev Saúde Pública. 2000; 34(Supl 6):62-72.

6. Zlotkin SH, Schauer C, Christofides A, Sharieff W, Tondeur MC, Hyder SM. Micronutrient sprinkles to control childhood anaemia. PLoS Med. 2005; 2(1):e1. Comment in: PLoS Med. 2005; 2(7):e188; author reply e202.

7. Beinner MA, Lamounier JA. Recent experience with fortification of foods and beverages with iron for the control of iron-deficiency anemia in Brazilian children. Food Nutr Bull. 2003; 24(3):268-74.

8. Berg A. The nutrition factor: its role in national development. Washington (DC): Institute for Policy Studies; 1984.

9. Raunhardt O, Bowley A. Mandatory food enrichment. Nutriview. 1996; 1(Supl):1-44.

10. Hurrell R, Bothwell T, Cook JD, Dary O, Davidsson $L$, Fairweather-Tait $S$, et al. The usefulness of elemental iron for cereal flour fortification: a sharing united states technology to aid in the improvement of nutrition-SUSTAIN Task Force report. Nutr Rev. 2002; 60(12):391-406.
11. Ré MI. Microencapsulação: em busca de produtos 'inteligentes'. Ciência Hoje. 2000; 27(162):24-9.

12. Ramos $M$, Stein LM. Desenvolvimento do comportamento alimentar infantil. J Pediatr (Rio de Janeiro). 2000; 76(Suppl 3):S229-37.

13. Addessi E, Galloway AT, Visalberghi E, Birch LL. Specific social influences on the acceptance of novel foods in 2-5-year-old children. Appetite. 2005; 45(3):264-71.

14. Birch LL, Fisher JA. Appetite and eating behavior in children. Pediatr Clin North Am. 1995; 42(4): 931-53.

15. Galloway AT, Fiorito LM, Francis LA, Birch LL. 'Finish your soup': counterproductive effects of pressuring children to eat on intake and affect. Appetite. 2006; 46(3):318-23.

16. Sullivan SA, Birch LL. Infant dietary experience and acceptance of solid foods. Pediatrics. 1994; 93(2):271-7.

17. Sari M, Bloem MW, de Pee S, Schultink WJ, Sastroamidjojo S. Effect of iron-fortified candies on the iron status of children aged 4-6 y in East Jakarta, Indonesia. Am J Clin Nutr. 2001; 73(6): 1034-9.

18. Silva MR. Efeito de uma bebida láctea fermentada e fortificada com ferro no estado nutricional de ferro em pré-escolares [dissertação]. Viçosa: Universidade Federal de Viçosa; 2000.

19. Paula RA, Fisberg M. The use of sugar fortified with iron tris-glycinate chelate in the prevention of iron deficiency anemia in preschool children. Arch Latinoam Nutr. 2001; 51(Suppl 1):54-9.

20. Brasil. Ministério da Saúde. ANVISA. Portaria $n^{\circ}$ 31, de 13 de janeiro de 1998. Aprova o regulamento técnico referente a Alimentos Adicionados de Nutrientes Essenciais [acesso 2007 nov 28]. Disponível em: <http//www.anvisa.gov.br/legis/ portarias/31_98.htm>.

21. Dean AG, Dean JA, Coulombier D, Brendel KA, Smith DC, Burton AH et al. Epi Info [computer program]. Version 6: a word processing, database, and statistics program for epidemiology on microcomputers. Atlanta (GA): Centers for Disease Control and Prevention; 1994.

22. World Health Organization. Physical status: the use and interpretation of anthropometry. Geneva: WHO; 1995. Technical Report Series n.854.

23. Beinner MA, Lamounier JA, Tomaz C. Effect of ironfortified drinking water of daycare facilities on the hemoglobin status of young children. J Am Coll Nutr. 2005; 24(2):107-14. 
24. Torres MA, Sato K, Novo NF, Queiroz SS. O leite fortificado no controle da anemia carencial ferropriva, em crianças matriculadas nas creches municipais da Grande São Paulo. Bol Soc Bras Hematol Hemoter. 1994; 16(166):221-7.

25. Fisberg M, Ferreira AMA, Silva JV, Kliamca PE, Cardoso R, Giorgini E, et al. Nova fórmula láctea infantil: papel na prevenção da anemia carencial. Pediatria. 1999; 21(1):48-59.

26. Almeida CA, Crott GC, Ricco RG, del Ciampo LA, Dutra de Oliveira JE, Cantolini A. Control of irondeficiency anaemia in Brazilian preschool children using iron-fortified orange juice. Nutr Res. 2003; 23(1):27-33.

27. Dutra de Oliveira JE, Ferreira JB, Vasconcellos VP, Marchini JS. Drinking water as an iron carrier to control anemia in preschool children in a day-care center. J Am Coll Nutr. 1994; 13(2):198-202.

28. Fisberg M, Velloso EP, Ribeiro RMS, Zuelo M, Braga $J A P$, Neto CS, et al. Anemia ferropriva e estratégias de fortificação com ferro aminoquelato. Pediatr Atual. 1998; 11(4):19-26.

29. Dutra de Oliveira JE, Scheid MM, Desai ID, Marchini S. Iron fortification of domestic drinking water to prevent anemia among low socioeconomic families in Brazil. Int J Food Nutr.1996; 47(3):213-9.

30. Dutra de Oliveira JE, de Almeida CA. Domestic drinking water-an effective way to prevent anemia among low socioeconomic families in Brazil. Food Nutr Bull. 2002; 23(3 Suppl):213-6.

31. Birch LL. Effects of peer models' food choices and eating behaviors on preschoolers food preferences. Child Dev. 1980; 51(2):489-96.

Recebido em: 7/11/2006

Versão final reapresentada em: 26/2/2008 Aprovado em: 25/9/2008 